\title{
Identifying rapidly parasiticidal anti-malarial drugs using a simple and reliable in vitro parasite viability fast assay
}

María Linares, Sara Viera, Benigno Crespo, Virginia Franco, María G. Gómez-Lorenzo, María Belén Jiménez-Díaz, Íñigo Angulo-Barturen, Laura María Sanz and Francisco-Javier Gamo*

\begin{abstract}
Background: The emergence of Plasmodium falciparum resistance to artemisinins threatens to undermine the effectiveness of artemisinin-based combination anti-malarial therapy. Developing suitable drugs to replace artemisinins requires the identification of new compounds that display rapid parasite killing kinetics. However, no current methods fully meet the requirements to screen large compound libraries for candidates with such properties. This study describes the development and validation of an in vitro parasite viability fast assay for identifying rapidly parasiticidal anti-malarial drugs.
\end{abstract}

Methods: Parasite killing kinetics were determined by first culturing unlabelled erythrocytes with $P$. falciparum in the presence of anti-malarial drugs for 24 or $48 \mathrm{~h}$. After removing the drug, samples were added to erythrocytes prelabelled with intracellular dye to allow their subsequent identification. The ability of viable parasites to re-establish infection in labelled erythrocytes could then be detected by two-colour flow cytometry after tagging of parasite DNA. Thus, double-stained erythrocytes (with the pre-labelled intracellular dye and the parasite DNA dye) result only after establishment of new infections by surviving parasites. The capacity of the test anti-malarial drugs to eliminate viable parasites within 24 or $48 \mathrm{~h}$ could, therefore, be determined.

Results: The parasite viability fast assay could be completed within $48 \mathrm{~h}$ following drug treatment and distinguished between rapidly parasiticidal anti-malarial drugs versus those acting more slowly. The assay was validated against ten standard anti-malarial agents with known properties and results correlated well with established methods. An abbreviated assay, suitable for adaption to medium-high throughput screening, was validated and applied against a set of 20 compounds retrieved from the publically available Medicines for Malaria Venture 'Malaria Box'.

Conclusion: The quantification of new infections to determine parasite viability offers important advantages over existing methods, and is amenable to medium-high throughput screening. In particular, the parasite viability fast assay allows discrimination of rapidly parasiticidal anti-malarial candidates.

Keywords: Drug discovery, Malaria, Parasiticidal, Plasmodium falciparum, Anti-malarial

\section{Background}

Plasmodium falciparum malaria remains a formidable global health challenge. Although there have been recent improvements in mortality, 584,000 deaths were

${ }^{*}$ Correspondence: fjg19447@gsk.com

R\&D Alternative Discovery and Development, Diseases of the Developing World, GlaxoSmithKline, 28760 Tres Cantos, Madrid, Spain attributable to this parasite in 2013, and most of the victims were children [1].

At the end of the last century, $P$. falciparum resistance to the most clinically important anti-malarial agents had become widespread. The discovery and introduction of artemisinin-based combination therapy (ACT) salvaged the effectiveness of anti-malarial therapy. However, delayed parasite clearance in response to ACT has been observed clinically, and the development of parasite 
resistance to these agents should be anticipated [2]. The loss of artemisinin efficacy would threaten recent advances in malaria mortality and morbidity and compromise the malaria eradication agenda [3].

Artemisinins are rapidly parasiticidal, quickly relieving malarial symptoms and minimizing the within-patient window of opportunity for resistant parasite selection and subsequent dissemination [4]. Replacement of artemisinins requires the identification of new drugs with similar rapid parasite killing kinetics. Ideally, such properties need to be identified in the early stages of the drug development process. However, no current methods fully meet the requirements to screen large compound libraries for rapidly parasiticidal anti-malarial drug candidates.

Standard in vitro parasite growth inhibition assays expose parasites to an anti-malarial drug for a defined period of time and determine the remaining viable parasites by labelling nucleic acids or measuring enzymatic activity. However, these methods do not allow direct determination of the rapidity of parasite killing-metabolism is not always a good surrogate of parasite viability, and the reliability of such tests depends on the drug mechanism of action [5, 6]. Recently, a number of different approaches have been proposed for the assessment of parasite viability after drug treatment [7-10]. However, none of these methods provide a complete time course for parasite killing over a broad range of anti-malarial compounds with a variety of different modes of action.

In 2012, a low-throughput assay was developed at Tres Cantos, based on the direct measurement of $P$. falciparum parasite viability in response to drug treatment over time. This methodology quantifies accurately the number of parasites able to resume a productive growth after removal of the drug and addition of fresh erythrocytes. The method is based on limiting serial dilutions of treated parasites and their culture for up to 28 days to enable wells with a single viable parasite to render detectable parasitaemia. This standardized assay allows establishment of different parameters, such as the in vitro parasite reduction ratio (PRR) and the presence of a lag phase in response to treatment with a specific anti-malarial. Nevertheless, this protocol is very labour intensive and time consuming and difficult to adapt as a higher throughput assay [5].

This study describes the development and validation of an in vitro parasite viability fast assay for identifying rapidly parasiticidal anti-malarial drugs. The methodology has been adapted from a previously described invasion assay [11]. Parasite killing kinetics were determined by first culturing unlabelled erythrocytes with $P$. falciparum in the presence of anti-malarial drugs. Immediately following drug removal (at either 24 or
$48 \mathrm{~h})$, new erythrocytes were added which had been pre-labelled with intracellular dyes allowing their subsequent identification. Following incubation for $48 \mathrm{~h}$, the ability of viable parasites to re-establish infection in labelled erythrocytes could then be detected by twocolour flow cytometry after labelling of parasite DNA, i.e., double-stained erythrocytes (with the pre-labelled intracellular dye and the parasite DNA dye) result only after establishment of new infections by surviving parasites. Thus, the capacity of the test anti-malarial drugs to eradicate viable parasites within 24 or $48 \mathrm{~h}$ could be determined.

Although different in vitro and in vivo assays have been established to measure re-invasion of Plasmodium parasites [11-13], this is the first time that such an assay has been used for determining the speed of parasite killing by anti-malarial compounds. This methodology provides a breakthrough over existing methods as it enables assessment of anti-malarial killing profiles $48 \mathrm{~h}$ following drug treatment and is amenable to automation. Thus, it should now be possible to interrogate large compound libraries to identify the next generation of anti-malarial drugs with artemisinin-like killing profiles [14-16].

\section{Methods}

The final protocol is described below; optimization and validation of the assay is described in the "Results".

\section{Chemicals and materials}

Standard anti-malarial drugs were provided by GlaxoSmithKline and Medicines for Malaria Venture, except azithromycin which was purchased from USP (Rockville, MD, USA). Stock solutions were prepared in DMSO at $10 \mathrm{mM}$. The final DMSO concentration used in the experiments $(<0.5 \%)$ has no inhibitory effect on parasite cultures. Carboxylfluorescein diacetate succinimidyl ester (CFDA-SE) was purchased from Molecular Probes (California, USA), Hoechst 33342 and glutaraldehyde solution were purchased from Sigma (St Louis, MO, USA).

\section{In vitro culture of Plasmodium falciparum parasites}

Plasmodium falciparum strain 3D7A was obtained from the Malaria Research and Reference Reagent Resource Center (MR4) and cultured using a modification of the method described by Trager et al. [17]. Briefly, parasites were cultured using RPMI-1640 supplemented with $0.5 \%$ Albumax and $150 \mu \mathrm{M}$ hypoxanthine at $2 \%$ haematocrit under an atmosphere of $90 \% \mathrm{~N}_{2}, 5 \% \mathrm{CO}_{2}, 5 \% \mathrm{O}_{2}$ at $37{ }^{\circ} \mathrm{C}$. Erythrocytes were obtained from the Spanish Red Cross Blood Bank. 


\section{Erythrocyte labelling}

Erythrocytes were labelled with CFDA-SE. The protocol for erythrocyte labelling was optimized based on modification of a standard procedure [11]. Optimal conditions were incubation of the required volume of erythrocytes at $1 \%$ haematocrit in RPMI 1640 with a CFDA-SE concentration of $10 \mu \mathrm{M}$ at $37^{\circ} \mathrm{C}$ for $2 \mathrm{~h}$. The suspension was washed with complete medium and the labelled erythrocytes re-suspended to $1 \%$ haematocrit with complete medium and incubated for $30 \mathrm{~min}$ at $37^{\circ} \mathrm{C}$. Finally, cells were washed twice with RPMI-1640. Labelled erythrocytes were diluted at $1 \%$ haematocrit and stored at $4{ }^{\circ} \mathrm{C}$ for up to $24 \mathrm{~h}$.

\section{Drug treatment and infection of labelled erythrocytes}

Assay methodology is summarized in Fig. 1. The conditions for the viability assay were chosen to mimic those used for standard $\mathrm{IC}_{50}$ determination ( $2 \%$ haematocrit, $0.5 \%$ parasitaemia with $\geq 80 \%$ ring stages) [5]. Asynchronous cultures were incubated under shaking conditions to avoid multiple infections per erythrocyte. A culture volume of $50 \mu \mathrm{L}$ per well with parasites at $4 \%$ haematocrit and $0.5 \%$ parasitaemia was dispensed into V-bottom, 96-well plates containing $50 \mu \mathrm{L}$ of complete media with previously diluted drugs prepared at $2 \times$ their final concentration to give a final volume of $100 \mu \mathrm{L}$ per well and a final drug concentration $10 \times$ the respective 50 \% inhibitory concentration for P. falciparum $\left(\mathrm{PfIC}_{50}\right)$; this concentration has been shown in previous experiments to avoid sub-optimal drug exposure [5]. For the experimental plates, infected erythrocytes were exposed to drug for 24 or $48 \mathrm{~h}$ using standard incubation techniques at $37{ }^{\circ} \mathrm{C}$. Drug was renewed every $24 \mathrm{~h}$ throughout the treatment period by retiring old media from the cultured wells and adding the same volume with fresh drug. Drug was removed following either 24 or $48 \mathrm{~h}$ of exposure. To remove drug, $80 \mu \mathrm{L}$ of media containing drug was removed, leaving a $20-\mu \mathrm{L}$ sample to which $200 \mu \mathrm{L}$ of fresh media was added (1/10 dilution). Plates were centrifuged for $10 \mathrm{~min}$ at $600 \mathrm{~g}$ (Beckman Allegra $\mathrm{X}-12 \mathrm{R})$, and $180 \mu \mathrm{L}$ of media then removed and the remaining $20 \mu \mathrm{L}$, containing the infected erythrocytes, was re-suspended in $100 \mu \mathrm{L}$ of complete media (1/5 dilution). Following drug removal, $70 \mu \mathrm{L}$ of washed infected erythrocytes was immediately transferred to a new microtitre plate containing $130 \mu \mathrm{L}$ of CFDA-SE-labelled, noninfected erythrocytes ( $1 / 3$ dilution) at $2 \%$ haematocrit in complete media. Plates were incubated for $48 \mathrm{~h}$ at $37^{\circ} \mathrm{C}$, $5 \% \mathrm{CO}_{2}, 5 \% \mathrm{O}_{2}$, and $90 \% \mathrm{~N}_{2}$ to allow new infections to develop in labelled erythrocytes as a surrogate measure of viable parasites. Control wells contained no drug and were exposed for $0 \mathrm{~h}$ before progression to the parasite invasion step using identical methods to those used for experimental plates.

\section{Parasite labelling}

Following incubation, parasite cultures were washed with phosphate buffer saline (PBS). Plates containing $200 \mu \mathrm{L}$ of cultured parasites were centrifuged and erythrocytes suspended with $2 \mu \mathrm{M}$ Hoechst 33342 in RPMI 1640 and incubated at $37^{\circ} \mathrm{C}$ for one hour in the dark. To allow the assay to be used for medium-high throughput screening,

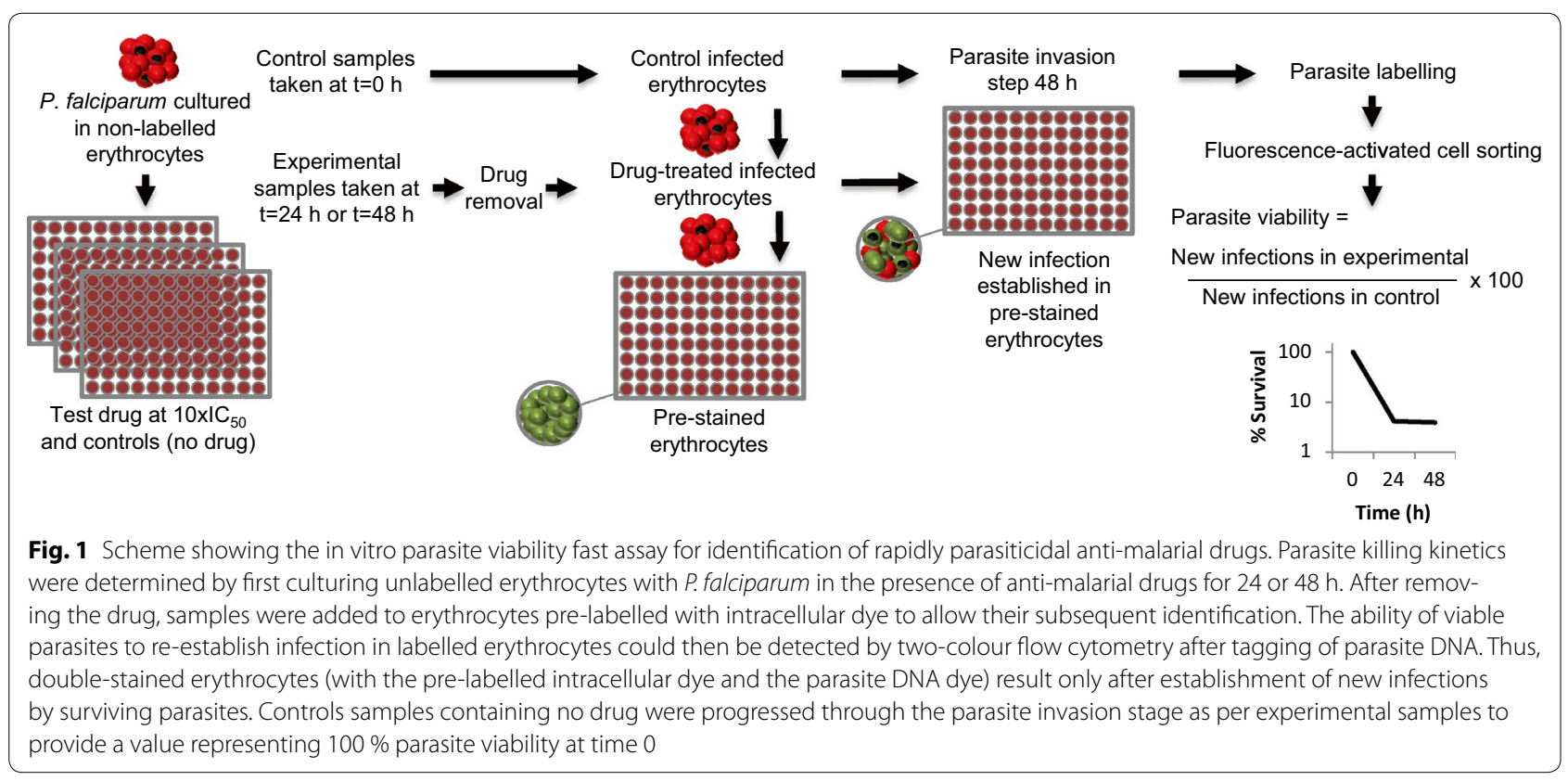


this method was modified by replacement of the medium with Hoechst solution $(200 \mu \mathrm{L})$ and an incubation of $20 \mathrm{~min}$. Cells were then fixed with $0.02 \%$ glutaraldehyde and stored at $4{ }^{\circ} \mathrm{C}$ protected from the light until flow cytometry analysis.

\section{Fluorescence-activated cell sorting (FACS)}

Following parasite staining, samples $(60 \mu \mathrm{L})$, were transferred to cytometry tubes containing $300 \mu \mathrm{L}$ of saline buffer (final haematocrit $0.3 \%$ ) and acquired in a LSRII flow cytometer [Becton-Dickinson (BD) Pharmingen, San Diego, CA, USA] equipped with a $355 \mathrm{~nm} 20 \mathrm{~mW}$ ultraviolet (UV) laser and a $488 \mathrm{~nm} 15 \mathrm{~mW}$ blue laser. Hoechst 33342 was excited by a UV laser and detected by a $450 / 40$ filter. CFDA-SE was excited by a blue laser and detected by a 530/30 filter. Samples were analysed using FACSDiva software (Becton-Dickinson). In those samples with parasitaemia $>0.1 \%$, the number of erythrocytes acquired was that which assured the gathering of at least 1000 events in the region established for infected erythrocytes. For parasitaemias $<0.1 \%, 8 \times 10^{5}$ total erythrocytes were acquired.

\section{Data analysis and validation}

Parasitized CFDA-SE-stained erythrocytes represent new infections that have been established from parasites surviving drug treatment. Thus, quantification of CFDASE-stained erythrocytes was used to evaluate parasite viability following drug treatment. In order to compare between different experiments, parasite viability was calculated as the percentage of infected CFDA-SE-stained erythrocytes in drug-treated samples at 24 or $48 \mathrm{~h}$ versus infected CFDA-SE-stained erythrocytes in concurrent control samples at time 0 , i.e., controls allow normalization to a parasite viability of $100 \%$ before drug treatment. The results were represented as the mean of this normalized percentage \pm the standard error of the mean.

All statistical analyses were performed using the GraphPad Prism Software (version 6). The non-parametric test U-Mann-Whitney was used to compare the two groups under comparison. Differences between means were considered significant when the $P$ value was $<0.05$.

\section{Results}

\section{Optimization of erythrocyte staining}

A previously described protocol for staining erythrocytes used erythrocytes labelled with $20 \mu \mathrm{M}$ CFDA-SE for $120 \mathrm{~min}$ at $2 \%$ haematocrit [11]. Optimization of this method tested decreasing concentrations of CFDA-SE $(20 \mu \mathrm{M}, 10 \mu \mathrm{M}$ and $5 \mu \mathrm{M})$ at 1 or $2 \%$ haematocrit. Concentrations of $10 \mu \mathrm{M}$ CFDA-SE were sufficient for staining erythrocytes at $1 \%$ haematocrit and enabled signal for detection (Fig. 2a) and labelling was stable for at least 7 days (Fig. 2b). Erythrocyte labelling incubation times were also tested $\left(30,60\right.$ and $120 \mathrm{~min}$ at $37^{\circ} \mathrm{C}$ ), with no significant differences in performance (Fig. 2c). Thus, a 30-min incubation time was selected.

\section{Estimating the rate of new infection by flow cytometry}

To confirm that the optimized labelling conditions could detect new infections, P. falciparum cultures with $0.5 \%$ parasitaemia were grown in the presence of labelled erythrocytes for $48 \mathrm{~h}$. The final concentration of labelled erythrocytes was two-thirds of the total amount in the culture.

Flow cytometry analysis revealed four different erythrocyte populations (Fig. 3a: non-infected non-stained erythrocytes $(\mathrm{RBC})$, infected non-stained erythrocytes (i-RBC), non-infected stained erythrocytes (RBC $\left.{ }^{C F D A-S E}\right)$ and infected stained erythrocytes (i-RBC $\left.{ }^{\mathrm{CFDA}-S \mathrm{~S}}\right)$. Although i-RBC could result from initial or new infections of non-stained erythrocytes, all instances of i-RBC ${ }^{C F D A-S E}$ must result from new infections. Thus, the quantification of $\mathrm{i}-\mathrm{RBC} \mathrm{C}^{\mathrm{CFDA}-\mathrm{SE}}$ can be used to determine parasite viability following drug treatment.

As previously described, there was no difference in the rate of infection between pre-stained and non-stained erythrocytes [11]. In fact, as can be seen in Fig. 3a, the ratio between $\mathrm{i}-\mathrm{RBC} \mathrm{CFDA}^{\mathrm{CFE}}$ and $\mathrm{i}-\mathrm{RBC}$ did not differ significantly from the ratio between $\mathrm{RBC}^{\mathrm{CFDA}-\mathrm{SE}}$ and $R B C$, indicative that both populations of erythrocytes ( $R B C^{\mathrm{CFDA}-S E}$ and $\mathrm{RBC}$ ) were equally susceptible to invasion. This is important because, as samples of treated, non-labelled erythrocytes are diluted three-fold by adding labelled erythrocytes, as much as two-thirds of the new infections can be quantified, but not the approximately one-third of infections that are produced in the initial, non-stained erythrocytes. As no bias was detected, the total number of new infections can be estimated from the quantified events, i.e., total infections $=3 / 2 \mathrm{i}-\mathrm{RBC}^{\mathrm{CFDA}-\mathrm{SE}}$.

\section{Dynamic range of the assay}

The dynamic range of the technique was estimated by growing serial dilutions of an initial P. falciparum 3D7A culture at $2 \%$ parasitaemia ( $2 \%$ haematocrit) in the presence of RBC CFDA-SE for $48 \mathrm{~h}$. As it is shown in Fig. 3b, established conditions allowed detection of initial parasitaemia levels as low as $0.002 \%$. Fluorescent quantification of $\mathrm{i}-\mathrm{RBC} \mathrm{CFDA}^{\mathrm{CEE}}$ was proportional to the number of initial parasites in the range of $0.5-0.005 \%$ parasitaemia. This dynamic range allows clear differentiation between fast-acting compounds and moderate/slow anti-malarial agents. 

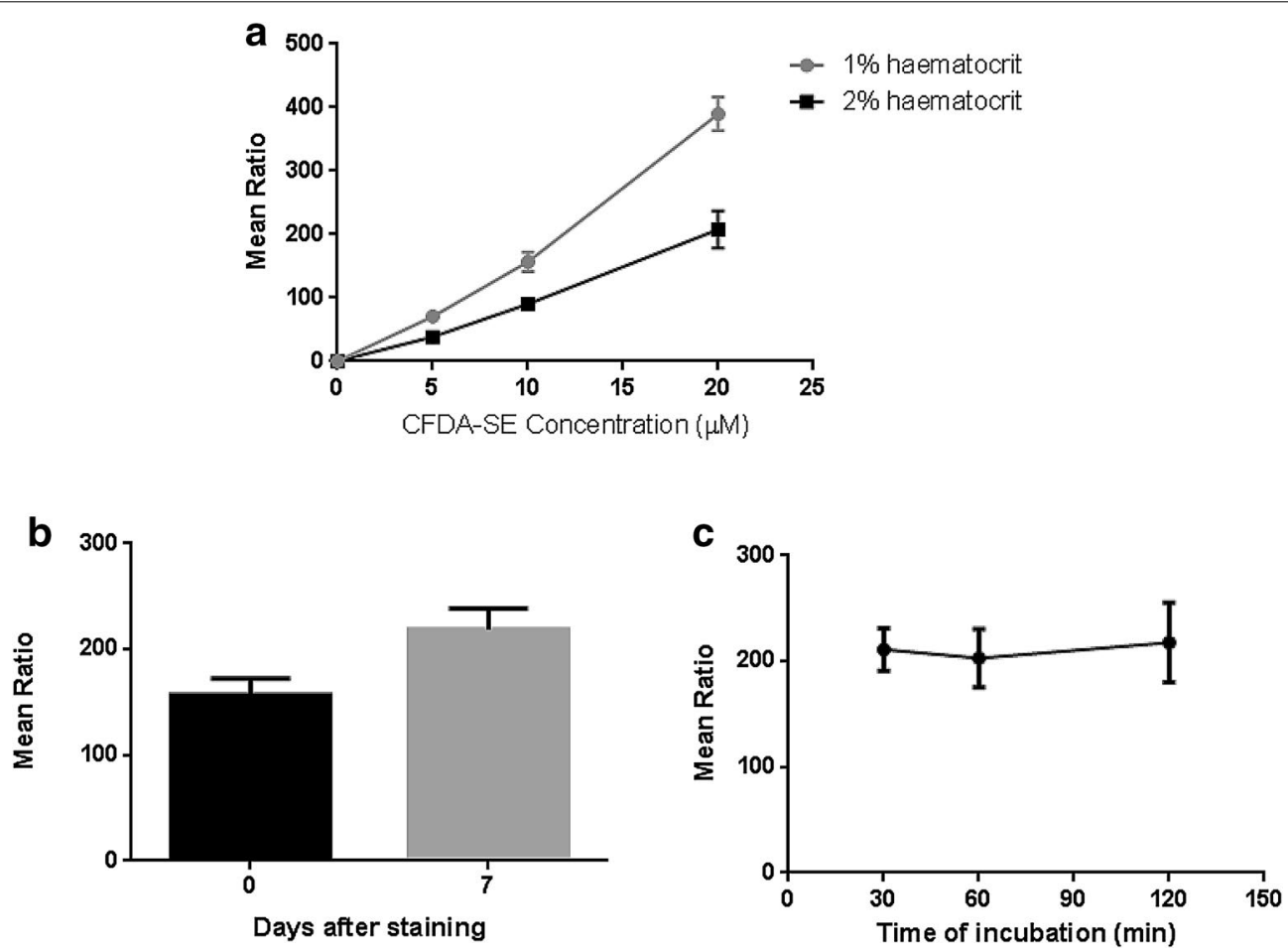

Fig. 2 Optimization of erythrocyte labelling for fluorescence-activated cell sorting detection. Graphs represent the ratios of mean fluorescence intensities from positive cells with respect to the negative population. Error bars represent SEM for three replicates. $\mathbf{a}$ Optimization of the assay used different concentrations of CFDA-SE (20, 10 and $5 \mu \mathrm{M})$, were tested to stain erythrocytes using 1 or $2 \%$ haematocrit. b Signal intensity by labelled erythrocytes remained robust for detection at least 7 days after staining (CFDA-SE $10 \mu \mathrm{M}, 1 \%$ haematocrit). c To test whether a reduction in incubation time could be achieved, different times (30 min, 1 and $2 \mathrm{~h}$ ) were used for staining erythrocytes with $10 \mu \mathrm{M} \mathrm{CFDA-SE}$ at $1 \%$ haematocrit
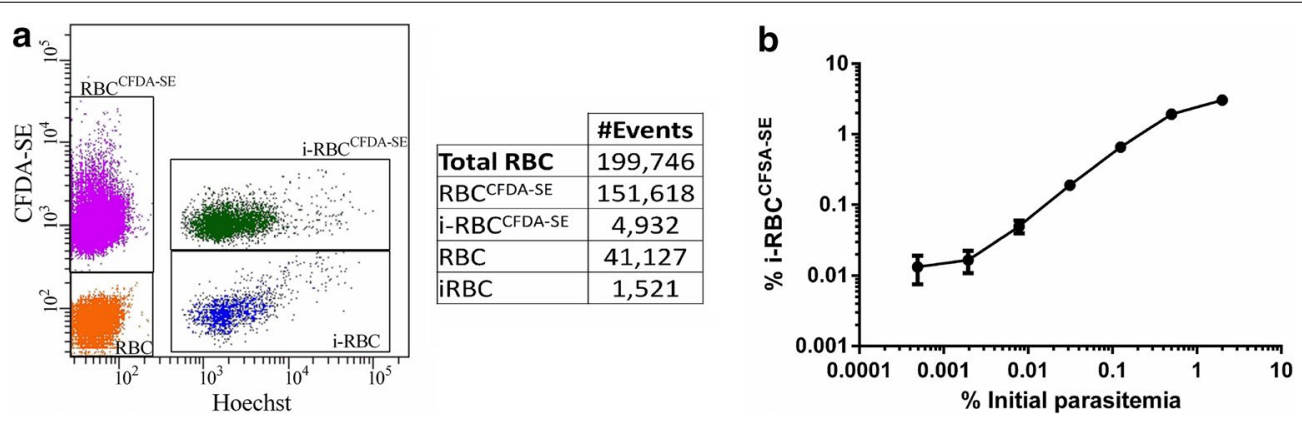

Fig. 3 Fluorescence-activated cell sorting (FACS) analysis of infected erythrocytes labelled with CFDA-SE. a DNA staining with Hoechst of parasites grown in the presence of CFDA-SE labelled erythrocytes allows robust detection of four distinct cellular populations. Data are representative of two independent experiments. b A P. falciparum 3D7 inoculum grown in standard culture conditions at $2 \%$ haematocrit and $2 \%$ parasitaemia was diluted (fourfold serial dilutions), in fresh media containing labelled non-infected erythrocytes for $48 \mathrm{~h}$. Parasites were labelled with Hoechst and fluorescence of i-RBC ${ }^{\text {CFDA-SE }}$ quantified by FACS. Y axis represents \% of i-RBC ${ }^{\text {CFDA-SE }}$ with respect to total number of RBC. Error bars represent SEM for three replicates. Established conditions allow detection of initial parasitaemia as low as $0.002 \%$

\section{Assay validation}

The assay was validated against four anti-malarial agents with well-characterized parasite killing profiles: atovaquone and pyrimethamine with a slow/moderate rate of killing and chloroquine and artesunate with rapid parasite killing. As shown in Fig. 4, the assay was able to distinguish between fast- and slow-acting agents within $48 \mathrm{~h}$ after drug treatment. Consequently, 24 and $48 \mathrm{~h}$ 


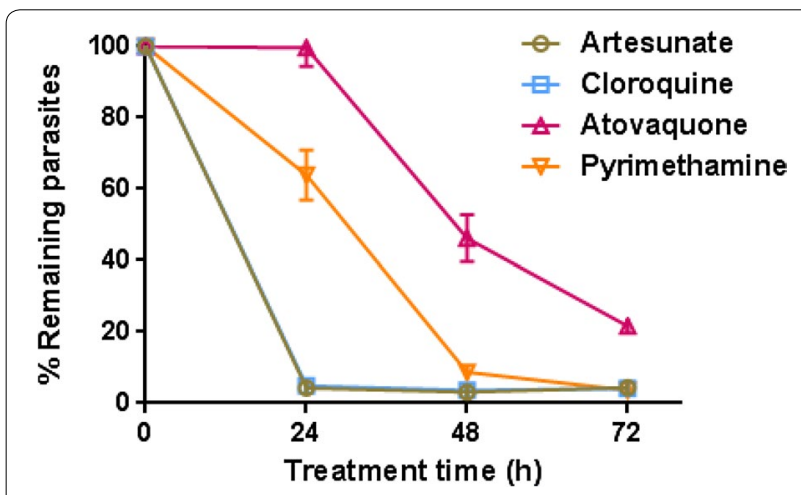

Fig. 4 Determination of killing rate profile for different classical antimalarial drugs. Plasmodium falciparum viability time-course profile for artesunate, chloroquine, atovaquone, and pyrimethamine at $10 \times$ $I_{50}$. Fast-acting anti-malarial drugs (artesunate and chloroquine) can be readily distinguished from moderate or slow-acting compounds (pyrimethamine and atovaquone). The number of viable infected erythrocytes was normalized using the number of untreated parasites. Error bars represent SEM for 4-9 replicates

were identified as the most relevant time points that could provide the information required to unequivocally identify rapidly parasiticidal compounds. These results correlate well with killing rate profiles reported using PRR methodology and equally can be attributed to the different anti-malarial modes of action tested [5].

\section{Adaptation for medium-high throughput screening}

This new methodology is more amenable for mediumhigh throughput than the standard PRR assay [5]. Killing profiles of different drugs can be determined in only $48 \mathrm{~h}$ after drug treatment instead of the 3-4 weeks required to detect single viable parasite using the limiting dilution method [5].

The following modifications were tested: (1) parasites were directly stained without removing the culture media, and, (2) culture media was replaced by Hoechst solution. Additionally, different incubation times (10, 15 and $20 \mathrm{~min}$ ) were tested before fixation. An incubation time of $20 \mathrm{~min}$ when the medium was replaced by Hoechst solution was selected as optimal, and used for subsequent experiments. Evaluation of atovaquone, pyrimethamine, chloroquine, and artemisinin using the abbreviated format produced similar results as for the initial format.

To further validate the abbreviated format, a panel of six additional standard anti-malarial molecules was also tested (Fig. 5). As expected, mefloquine, piperaquine, lumefantrine, halofantrine, and pyronaridine showed a fast-acting profile. However, azithromycin did not show any effect on parasite viability, consistent with its mode of action: azithromycin affects protein synthesis in the

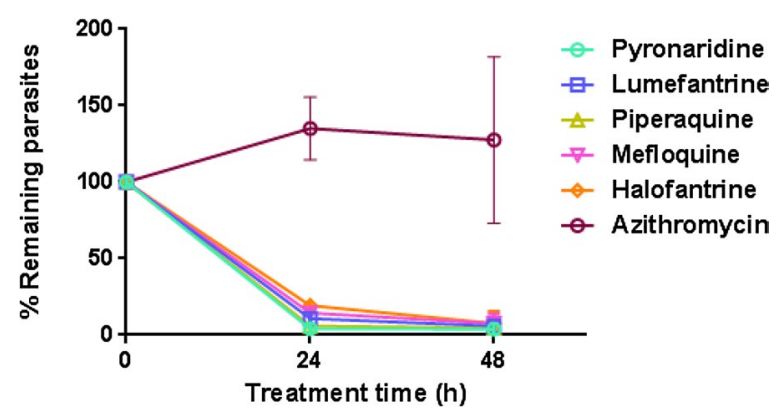

Fig. 5 Two-time point viability analysis using an abbreviated procedure. Plasmodium falciparum cultures were treated with indicated drugs and parasites sampled after 24 and $48 \mathrm{~h}$. Medium-throughput conditions allowed discrimination of fast-acting profiles of pyronaridine, halofantrine, lumefantrine, piperaquine and mefloquine from the azithromycin anti-malarial profile, with parasite killing only in the second generation. The number of viable infected erythrocytes was normalized using the number of untreated parasites. Error bars represent SEM for 4-9 replicates

Plasmodium apicoplast and anti-malarial effects are only observed after two cycles of intra-erythrocytic development [18].

Furthermore, the abbreviated assay was further validated and applied to identify novel rapidly parasiticidal anti-malarial agents. A set of 20 compounds retrieved from the Medicines for Malaria Venture 'Malaria box' [19], was tested with the parasite viability fast assay and the previously described in vitro killing rate assay using limiting dilutions [5]. The profiles of rapidly parasiticidal anti-malarial agents (chloroquine/artemisinin-like) were clearly differentiated from those of slow (atovaquonelike) or moderate (pyrimethamine-like) compounds and results were confirmed independently of the method used (Table 1). None of the compounds that displayed a fast parasite killing profile was previously known to have this property. Thus, these potentially represent new avenues of investigation for the development of novel anti-malarial agents which have parasite killing kinetics similar to artemisinin.

\section{Discussion}

Artemisinin resistance is emerging and threatens to undermine the effectiveness of anti-malarial therapy. Consequently, identifying anti-malarial drugs that could succeed artemisinins, and which retain their key property of rapid killing of Plasmodium is a priority.

The parasite viability fast assay described in this paper is an important step to enable the identification of rapidly parasiticidal anti-malarial agents. Recent efforts have identified numerous leads for new anti-malarial agents [14-16]. However, their evaluation for artemisinin-like parasite killing kinetics has been limited, as current 
Table 1 Comparison between the parasite viability kinetic assay and the in vitro killing rate assay based on limiting dilutions using compounds from the Medicines for Malaria Venture Malaria Box

\begin{tabular}{|c|c|c|}
\hline Compound & $\begin{array}{l}\text { In vitro killing rate } \\
\text { assay (standard assay) }\end{array}$ & $\begin{array}{l}\text { Viability assay } \\
\text { (fast assay) }\end{array}$ \\
\hline MMV000478 & Fast & Fast \\
\hline MMV665939 & Slow & Slow \\
\hline MMV019066 & Slow & Slow \\
\hline MMV019662 & Slow & Slow \\
\hline MMV007564 & Moderate & Fast/moderate \\
\hline MMV020750 & Moderate & Moderate \\
\hline MMV665789 & Moderate & Moderate \\
\hline MMV665928 & Fast & Fast \\
\hline MMV006767 & Moderate & Moderate \\
\hline MMV008149 & Slow & Slow \\
\hline MMV009108 & Slow & Slow \\
\hline MMV665924 & Fast & Fast \\
\hline MMV011895 & Fast & Fast \\
\hline MMV019017 & Fast & Fast \\
\hline MMV009063 & Fast & Fast \\
\hline MMV665794 & Moderate & Moderate \\
\hline MMV665824 & Slow & Slow \\
\hline MMV006455 & Fast & Fast \\
\hline MMV665852 & Moderate/fast & Fast \\
\hline MMV665888 & Moderate & Moderate \\
\hline
\end{tabular}

Fast chloroquine/artemisinin-like (no viable parasites detected after $24 \mathrm{~h}^{\prime}$ treatment), moderate pyrimethamine-like (no viable parasites detected after $48 \mathrm{~h}$ ' treatment), slow atovaquone-like (more than $48 \mathrm{~h}$ ' treatment needed to kill all parasites)

methods are time consuming and cannot easily be scaled to screen the thousands of candidate molecules.

The parasite viability fast assay described here was able to distinguish between anti-malarial agents with artemisinin-like parasite killing kinetics and those that act more slowly within $48 \mathrm{~h}$ following drug treatment. The assay was validated against standard anti-malarial drugs with known properties and results correlated well with established methods.

The availability of a medium-high throughput assay for identifying compounds with artemisinin-like parasite killing kinetics is a critical step in developing the next generation of anti-malarial therapy. Thus, the assay described here has been adapted to allow its application in medium-high throughput screening and has been evaluated against a test set of candidate compounds. The methodology lends itself to automation, using simple incubations and washing steps without the labourintensive procedures used for other assays, such as the limiting dilution method. Staining stability allows storage, and multiple samples can be simultaneously assayed.
It is envisaged that the assay could be used for screening compound libraries such as the Tres Cantos Antimalarial Set (TCAMS), which includes nearly 13,500 compounds. The parasite viability kinetic assay could be used with any laboratory parasite strain, including adapted ex vivo isolates. This could be particularly relevant in the evaluation of artesunate derivatives, as resistant parasites do not present any change in terms of in vitro $\mathrm{IC}_{50}$, and complex read-outs are required [20,21].

The parasite viability fast assay described here has a less sensitive limit of detection (equivalent to $0.002 \%$ parasitaemia) compared with the limiting dilution method (equivalent to $0.000005 \%$ parasitaemia) [5]. Thus, any compound that elicited a decrease in parasitaemia of $>99 \%$ would have the same profile in the assay. Although this limit of detection is sufficient to differentiate between fast-acting compounds and moderate/slow-acting ones, it cannot resolve differences between two fast-acting compounds (e.g., artemisinin and chloroquine). A further limitation of the methodology is that it cannot evaluate the parasiticidal effects of compounds that display a delayed death phenotype, for example, azithromycin. However, molecules with such a mechanism of action can be identified using standard evaluations at 48 and $96 \mathrm{~h}$ to prove a shift in the $\mathrm{IC}_{50}$. Alternatively, the methodology described here could be adapted by extending the incubation time with prelabelled erythrocytes to cover two parasite lifecycles.

The parasite viability fast assay offers a rapid, direct measurement of parasite killing that does not rely on parasite metabolic activity or accumulation of specific molecules. Thus, potential bias or artefacts that appear when viability and parasite metabolic activity become uncoupled are avoided. Although other assays have been developed for determining parasite killing kinetics, they use indirect measurements to assess parasite viability [8, 9]. For example, the rapid assay described by Le Manach uses a modification of the standard $\left[{ }^{3} \mathrm{H}\right]$ hypoxanthine incorporation assay for $\mathrm{IC}_{50}$ calculations [9]. However, atovaquone and pyrimethamine do not display the expected profile corresponding to their mechanism of action [9]. A method that detects shifts in parasite mRNA levels has been described by Bahamontes-Rosa [8], but can only differentiate between cidal or static behaviours and cannot evaluate the speed of parasite killing. The method is also laborious and time consuming, reporting results at $240 \mathrm{~h}$ post-treatment [8].

Exceptional efforts have greatly expanded the pool of potential candidate molecules with anti-plasmodial activity [14-16]. The main challenge now is to rapidly process those candidates to identify the next generation of anti-malarial therapy. The parasite viability fast assay described here is robust, reliable, rapid, expandable, and 
could be easily automated. The methodology requires no specialized equipment and can be easily implemented in standard laboratories. The assay can also be used with different parasite strains, offering a flexible research tool. The quantification of new infections to assess parasite viability offers important advantages over existing methods, and in particular allows discrimination of rapidly parasiticidal anti-malarial candidates that have the potential to succeed the artemisinins.

\section{Authors' contributions}

$M L$ carried out the in vitro experiments, participated in the design of the study and performed the statistical analysis. SV carried out the analysis of cytometry. $B C$ helped to interpret the data. VF and MGGL participated in the validation of the assay. MBJD and IA participated in the design of the study. LMS and FJG conceived of the study, participated in its design and coordination and helped to draft the manuscript. All authors read and approved the final manuscript.

\section{Acknowledgements}

We would like to thank José Luis Llergo-Largo and Cristina De Cózar for the help with all in vitro experiments. Naomi Richardson of Magenta Communication Ltd. provided editorial assistance and was funded by GlaxoSmithKline. This study was funded by GlaxoSmithKline.

\section{Competing interests}

All authors are employees of GlaxoSmithKline and SV, MGGL, MBJD, IA, LMS and FJG own shares of the company.

Received: 23 June 2015 Accepted: 22 October 2015

Published online: 05 November 2015

\section{References}

1. WHO. World Malaria Report. World Health Organization, Geneva. 2014. [http://www.who.int/malaria/publications/world_malaria_report_2014/ wmr-2014-no-profiles.pdf?ua=1]. Accessed on 29 Mar 2015.

2. Dondorp AM, Fairhurst RM, Slutsker L, MacArthur JR, Breman JG, Guérin PJ, et al. The threat of artemisinin-resistant malaria. N Engl J Med. 2011;365:1073-5.

3. Lubell Y, Dondorp A, Guérin PJ, Drake T, Meek S, Ashley E, et al. Artemisinin resistance-modelling the potential human and economic costs. Malar J. 2014;13:452.

4. Burrows JN, van Huijsduijnen RH, Mohrle JJ, Oeuvray C, Wells TN. Designing the next generation of medicines for malaria control and eradication. Malar J. 2013;12:187.

5. Sanz LM, Crespo B, De-Cozar C, Ding XC, Llergo JL, Burrows JN, et al. P. falciparum in vitro killing rates allow to discriminate between different antimalarial mode-of-action. PLoS One. 2012;7:e30949.
6. Wein S, Maynadier M, Van Tran BC, Cerdan R, Peyrottes S, Fraisse L, et al. Reliability of antimalarial sensitivity tests depends on drug mechanisms of action. J Clin Microbiol. 2010;48:1651-60.

7. Young RD, Rathod PK. Clonal viability measurements on Plasmodium falciparum to assess in vitro schizonticidal activity of leupeptin, chloroquine, and 5-fluoroorotate. Antimicrob Agents Chemother. 1993;37:1102-7.

8. Bahamontes-Rosa N, Rodriguez-Alejandre A, Gonzalez-del-Rio R, GarciaBustos JF, Mendoza-Losana A. A new molecular approach for cidal vs static antimalarial determination by quantifying mRNA levels. Mol Biochem Parasitol. 2012;181:171-7.

9. Le Manach C, Scheurer C, Sax S, Schleiferbock S, Cabrera DG, Younis Y, et al. Fast in vitro methods to determine the speed of action and the stage-specificity of anti-malarials in Plasmodium falciparum. Malar J. 2013;12:424.

10. Painter HJ, Morrisey JM, Vaidya AB. Mitochondrial electron transport inhibition and viability of intraerythrocytic Plasmodium falciparum. Antimicrob Agents Chemother. 2010;54:5281-7.

11. Theron M, Hesketh RL, Subramanian S, Rayner JC. An adaptable two-color flow cytometric assay to quantitate the invasion of erythrocytes by Plasmodium falciparum parasites. Cytometry A. 2010;77:1067-74.

12. Varela ML, Razakandrainibe R, Aldebert D, Barale JC, Jambou R. Cytometric measurement of in vitro inhibition of Plasmodium falciparum field isolates by drugs: a new approach for re-invasion inhibition study. Malar J. 2014;13:110.

13. Lelliott PM, Lampkin S, McMorran BJ, Foote SJ, Burgio G. A flow cytometric assay to quantify invasion of red blood cells by rodent Plasmodium parasites in vivo. Malar J. 2014;13:100

14. Plouffe D, Brinker A, McNamara C, Henson K, Kato N, Kuhen K, et al. In silico activity profiling reveals the mechanism of action of antimalarials discovered in a high-throughput screen. Proc Natl Acad Sci U S A. 2008;105:9059-64.

15. Gamo F-J, Sanz LM, Vidal J, de Cozar C, Alvarez E, Lavandera J-L, et al. Thousands of chemical starting points for antimalarial lead identification. Nature. 2010;465:305-10.

16. Guiguemde WA, Shelat AA, Bouck D, Duffy S, Crowther GJ, Davis PH, et al. Chemical genetics of Plasmodium falciparum. Nature. 2010;465:311-5.

17. Trager W, Jensen JB. Human malaria parasites in continuous culture. Science. 1976;193:673-5.

18. Yeo $\mathrm{AE}$, Rieckmann $\mathrm{KH}$. Increased antimalarial activity of azithromycin during prolonged exposure of Plasmodium falciparum in vitro. Int J Parasitol. 1995;25:531-2.

19. Spangenberg T, Burrows JN, Kowalczyk P, McDonald S, Wells TN, Willis P. The open access malaria box: a drug discovery catalyst for neglected diseases. PLoS One. 2013;8:e62906.

20. Witkowski B, Amaratunga C, Khim N, Sreng S, Chim P, Kim S, et al. Novel phenotypic assays for the detection of artemisinin-resistant Plasmodium falciparum malaria in Cambodia: in vitro and ex vivo drug-response studies. Lancet Infect Dis. 2013;13:1043-9.

21. Amaratunga C, Neal AT, Fairhurst RM. Flow cytometry-based analysis of artemisinin-resistant Plasmodium falciparum in the ring-stage survival assay. Antimicrob Agents Chemother. 2014;58:4938-40.

\section{Submit your next manuscript to BioMed Central and take full advantage of:}

- Convenient online submission

- Thorough peer review

- No space constraints or color figure charges

- Immediate publication on acceptance

- Inclusion in PubMed, CAS, Scopus and Google Scholar

- Research which is freely available for redistribution

Submit your manuscript at www.biomedcentral.com/submit
() Biomed Central 